\title{
EXPERIENCE AND RESULTS OF SURGICAL TREATMENT OF PATIENTS WITH CARDIAC NEOPLASMS
}

\author{
Vlasko G.S. ${ }^{1}$, Lukin O.P. ${ }^{1,2}$, Belov D.V.1,2 \\ I"South-Ural State Medical University" of the Ministry of Healthcare of the Russian Federation, Chelyabinsk, e-mail: \\ Vlasko13@yandex.ru; \\ ${ }^{2}$ "Cardiovascular surgeon of the Russian Federal Center of Cardiovascular Surgery», Chelyabinsk, e-mail: \\ luk.o.p@mail.ru
}

Aims.

To study the clinical manifestations of cardiac tumours, single out the set of symptoms that suggest a neoplasm, to optimize the patient management approach at the stages of diagnosis and treatment.

Materials and methods.

In the period from 2011 to 2018,52 patients with cardiac tumours were operated on at Chelyabinsk FCVSC [Federal Cardiovascular Surgery Centre]. Neoplastic lesions of the left atrium were detected in 46 patients. Of these tumours of the left atrium, no obstruction of atrioventricular orifice was detected in 28 patients, and obstruction of atrioventricular orifice was detected in 18 patients. Neoplastic lesions of the right atrium were detected in 3 patients. In one case the following was found: neoplastic lesion in the left ventricular cavity, pericardial tumour, and extended tumour with infiltrative growth. The majority of patients were diagnosed with cardiac myxoma.

Results.

The analysis of obtained data revealed the diversity and polymorphous nature of the clinic picture of cardiac tumours, which more often depends on the tumour location rather than its histological structure. When the set of symptoms was singled out, the clinical picture of heart disease simulation with the development of congestive cardiovascular failure was detected in most patients.

Conclusions.

Tumours that are small but cause blood flow obstruction have earlier clinical manifestations compared to large tumours with infiltrative growth. For the purpose of early diagnosis of heart diseases, including cardiac tumours, screening tests (echocardiography) should be implemented when indicated in patients who have reached 40 years of age. Intraoperative histological examination of the tumour makes it possible to decide on the extent of surgical intervention and predict the risk of tumour recurrence.

Keywords: cardiac tumours, myxoma, malignant cardiac neoplasms, diagnosis of cardiac tumours, surgical treatment of cardiac tumours.

\section{INTRODUCTION.}

Cardiac tumors are pathological myocardial and pericardial neoplasms, the genetic mutations wherein disrupt cell growth and differentiation. Detecting a heart tumor might be difficult, as they have low-specific clinical signs [1;2]. Given the clinical polymorphism, this pathology is often found by chance when examining the cardiovascular system, or posthumously. Primary tumors can be both benign and malignant; they initially develop in the myocardium or in the pericardium, and are rare due to the myocardial metabolism, fast blood flow, and the limited lymphatic connectivity of the heart. Secondary tumors are metastases of extracardiac tumors [3]. The clinical course and prognosis might not actually correlate to the histological classification into benign and malignant tumors. Although benign, exophytic tumors pose a high risk of severe, often lethal thromboembolism [1;2]; $\sim 25 \%$ of cardiac tumors are malignant. Secondary tumors are the product of blood or lymph vesselmediated metastasis, although they may also appear due to the tumors invading from heart-adjacent organs. There are three core forms of metastatic heart malignancies: micronodular, macronodular, and diffuse infiltrative metastases [4]. Late diagnosis of cardiac tumors often results in the ineffective treatment of primary and secondary malignancies; such treatment rarely leads to a favorable long- 
term prognosis. However, the clinical experience of today, state-of-the-art diagnosis, and advances of cardiac surgery help detect heart tumors in time, which in turn lets consider them a likely curable pathology.

The research objectives hereof were: 1 . To analyze the records of cardiac tumor surgeries at the Federal Cardiovascular Surgery Center, Chelyabinsk. 2. To study the clinical manifestations of cardiac tumors so as to identify the complex of symptoms indicative of a neoplasm. 3 . To optimize patient management for diagnosis and treatment.

MATERIALS AND METHODS. From 2011 to 2018, the FCSC in Chelyabinsk operated 52 patients with cardiac tumors. Of them, 13 patients (25\%) were male, 39 (75\%) were female. The patients were aged 34 to 78 , or 59.6 on average.

The study protocol followed guidelines for experimental investigation with human subjects in accordance with the Declaration of Helsinki and was approved by the ethics committee. Written informed consent was obtained from each patient (or an official representative) before the study.

The following complaints and symptoms were registered at the primary doctor's visit: shortness of breath without excessive physical activity or when changing the body position, and stronger dyspnea in a horizontal position in 42 patients (80\%); tachycardia in 9 patients (18\%); chest discomfort and crushing pain behind the sternum unrelated to physical exercise in 10 patients (19.2\%); higher blood pressure (BP) in 5 patients (9.6\%); dry cough in 5 patients (9.6\%); productive cough in 3 patients (5.8\%); hemoptysis in 1 patient (1.9\%); low-grade fever in 2 patients (3.8\%); darkened vision, fainting, and dizziness in 5 patients $(9.6 \%)$; left-hand paresthesia and burning pain in the superior abdomen in 1 patient each $(1.9 \%)$; general weakness and increased fatigue in 6 patients (11.5\%); lower-limb edema in 1 patient (1.9\%). One patient had a tumor in the medical history (thigh liposarcoma). Clinical manifestations were associated with cardiac arrhythmia and conduction disorder in 16 patients (30.7\%); cerebral complications in the form of cerebrovascular accidents (CVA) in 4 patients (7.7\%); hypertension in 26 patients (50\%); congestive heart failure in 17 patients (32.7\%); pulmonary hypertension in 5 patients $(9.6 \%)$; morphological and functional changes in heart valves in 4 patients $(7.7 \%)$; afflicted coronary arteries (stenocardia, myocardial infarction) in 6 patients $(11.5 \%)$. All the patients underwent extensive examination that comprised echocardiography (echo), electrocardiography (ECG), chest $\mathrm{X}$-ray, and routine laboratory tests. Two patients had tumor recurrence. Distribution by localization was as follows: 46 patients or $88.6 \%$ had tumors in the left atrium (LA), 28 or $54.1 \%$ of the LA tumor patients had a non-obstructed atrioventricular (AV) valve, while 18 (34.5\%) had an obstructed one. Right-atrium (RA) tumors were found in 3 patients (5.7\%). Clinicians also identified (one case of each) a left ventricular (LV) cavity tumor, a pericardial cyst, and an elongated tumor on the anterior-superior heart surface involving the anterior LV wall, the interventricular septum, the right ventricular outflow tract, which affected the pericardium along the LV wall up to the heart top. Histological testing verified myxoma in 48 cases (92.3\%), pericardial cyst with RA compression in 1 case (1.9\%), and malignant heart tumors (MHT) in 3 patients, or $5.7 \%$. 
RESULTS. The approach was median sternotomy in 50 patients, sternotomy of the old suture in 2 patients who had a recurrent heart tumor, and thoracotomy in 2 patients. Fifty-one patients had artificial circulation and cold pharmacological cardioplegia during surgery. In one case, no artificial circulation and cold pharmacological cardioplegia were used. Isolated en block tumor removal was performed for 26 patients (50\%), in whom the tumor site was excised, and the appearing defect was sutured. In 2 patients, LA tumor removal was complemented with LA auricle ligation; pericardial cyst was removed from 1 patient (1.9\%). Where the tumor had destroyed the valve leaflets, they were repaired in $6(11.5 \%)$ patients using De Vega surgery if the tricuspid valve was damaged, or a MedInzh support ring if the mitral valve was damaged; the interatrial septum was repaired with a xenopericardial patch in $6(11.5 \%)$ patients. In 2 patients, LA tumor removal required surgical correction of the coronary flow. Tumor resection was performed in 6 cases $(11.5 \%)$. The hospital mortality rate totaled $1.9 \%$ or 1 patient; $90 \%$ of the patients saw significant improvement or full recovery.

Discussion. Data should be analyzed in the light of the diverse, polymorphic, and often nonspecific clinical signs of heart tumors. Sex-wise, women prevailed. The medical history contained a variety of complaints regardless of the tumor type, location, or degree of penetration into adjacent tissue. Two patients had paraneoplastic syndrome with low-grade fever, anemia, loss of appetite, reduced tolerance to exercising, and weight loss. According to the authors' data, the clinical signs of cardiac neoplasms depend not so much on the tumor histology as on the volume and anatomical location. Speaking of the symptom complex, most patients had a clinical picture of imitated heart malformations with a congestive cardiovascular failure. A mobile tumor or one localized in close proximity to $\mathrm{AV}$ valves might have clinical and hemodynamic signs reminiscent of stenosis or valve failure. Stenosis signs are more characteristic of tumors proximal to AV valves, while failure signs are more typical of a distal location that affects both the leaflets and the valve system. According to the authors' data, patients with left-chamber tumors were more likely to have congestive heart failure symptoms of the pulmonary circulation, as well as left ventricular failure signs. Besides, LA tumors were associated with LA hypertrophy signs in ECG. In MHT 3 patients, tumors long had the clinical signs of congestive heart failure on top of hydrothorax. One patient with a right-atrial tumor had symptoms of right-ventricular failure and systemic circulation congestions resulting in lower-limb edema. Of the operated patients, 11 had syncopal conditions and lipothymia in the history, which was likely caused by the transient mechanical blood flow obstruction in heart cavities when repositioning the body. Literature suggests that large-sized tumors in the heart cavity might disrupt hemodynamics and obstruct the blood flow at the valve level. They might also cause a dilation of the affected chamber by displacing a significant amount of blood while not obstructing the flow. In turn, the vertical position of the patient's body often disrupts hemodynamics as gravity pulls a mobile tumor down. This clinical picture is typical of myxomas localized in the atria [1]. Among the operated patients covered herein, $92.3 \%$ had a heart 
myxoma. Myxoma was most frequently localized in the LA cavity (44 patients or $84.6 \%$ ), rarely in the RA cavity (3 patients or 5.7\%), and extremely rarely in the LV cavity (1 patient or 1.9\%). Myxoma is a tumor the cells of which originate from a multipotent mesenchymal cell [3]. In most cases, myxoma is attached to subendothelial layers with a fibrovascular leg, rarely sitting on a wide foot. A myxoma can be smooth, encapsulated, or have a lobular polypoid structure. Gelatin myxomas are prone to embolization. Some tumors might have sites of hemorrhage, calcinosis, or cysts. The data of this research suggests that in 4 patients, myxoma manifested itself in the form of cerebral complications (ischemic cerebral infarction); other possible causes for stroke were excluded. Cardiac tumors are often diagnosed after CVA, embolisms in the peripheral vascular network, or pulmonary artery embolism caused by tumor fragmentation or thrombus mobilization [6]. Tumor tissue has an increased concentration of tissue thromboplastin, which causes the thrombotic mass to emerge on the tumor surface, resulting in systemic embolic complications. Blood flow- or body repositioning-induced mechanical deformation of, and injury to, the tumor facilitates the destruction of soft tumor surface and its fragmentation [1]. According to the authors' data, embolic complications are observed in $40 \%$ to $50 \%$ of all myxoma cases. Left-compartment myxomas are associated with systemic circulation embolism from the aorta to smaller intraorgan branches; cerebral arteries are embolized in 50\% of all cases, resulting in a short loss of consciousness. Later intracranial arterial embolism might lead to aneurysms and their rupture. Retinal artery embolism might cause transient or persistent loss of vision. Right-compartment myxoma might cause pulmonary artery embolism, sometimes leading to chronic post-embolic pulmonary hypertension. There are reports that histological testing of the removed embolus might detect heart myxoma, which has been confirmed surgically [1; 7]. As myxomas mainly occur in the LA, embolic complications mostly affect the systemic circulation. Two patients had tumor recurrence. Tumor resection was performed in 6 cases (11.5\%). A malignant invasive tumor had earlier been found in the thigh liposarcoma patient. She had her altered pericardium and a tumor fragment resected. Beside tumor growth in the heart chamber cavity, it can invade the adjacent tissue. Such invasion has no clear clinical signs and manifests itself as a dysfunction of the heart component it affects, i.e. compresses or displaces. Myocardial invasion that involves all the layers of the cardiac wall is a typical situation. Thus, heart tumors are clinically critical, albeit rare, cardiac diseases. Monitoring has shown that a benign cardiac tumor might take three or more years since its onset to diagnose. Echocardiography can actually suffice for diagnosis; however, tumor-related changes might be misinterpreted as artifacts if a tumor is not suspected. The method has technical limitations, too: it is difficult to examine the right chambers, and it has limited use for differential diagnosis of the pericardium. However, echo is highly available, safe, can be used for prolonged monitoring, and uses relatively inexpensive equipment, which makes it suitable for screening, as well as for noninvasive diagnosis of a reasonably suspected cardiac tumor [1]. The extent of surgical intervention is an important part of the surgeon's tactics. Intramuscular tumors are often 
only partly removable, meaning that only some fragments of the inflow- and outflow-obstructing tumor can be extracted. Cardiac tumor surgery must be designed not only for total tumor resection, but also with heart hemodynamics recovery in mind. Post-diagnosis survival ranges from 7 months to 2 years [6]. In the case of diffuse-infiltrative growth of tumors or malignancies in the heart, or if the tumor is large, extensive surgery might be required to simultaneously reconstruct the heart anatomy and chambers, implant valve prosthetics, restore the coronary flow, and implant a permanent pacemaker; all of this increases the operative risks and affects hospital mortality rates, survival, and duration of life $[4 ; 6 ; 8 ; 9]$.

Optimistic long-term postoperative prognosis requires intraoperative histological testing of the tumor to decide how extensive the surgery must be, as well as to predict the risks of recurrence. The authors hereof believe implanting an artificial heart or a heart transplant, the last recourse provided no metastases, is a promising solution [6].

\section{CONCLUSIONS.}

1. The clinical signs of cardiac tumors mainly depend on the tumor location rather than histology.

2. The clinical picture is polymorphous, and there are no specific symptoms that could help detect intracavity neoplasms of the heart, resulting in delayed diagnosis; however, even if diagnosed late, further surgery might improve the quality of life.

3. Early diagnosis of cardiac diseases including tumors requires indication-based echo screening of all patients aged 40 or older.

4. Small obstructive tumors manifest themselves earlier than larger invasive ones.

5. All intracavity heart tumors might affect multiple anatomical regions and pose the patient at risk of fatal complications (heart failure, arrhythmia, pericarditis, cardiac tamponade, systemic embolism, or cerebral complications), which is why surgical intervention is urgent.

6. Intraoperative histological testing helps decide on the extent of surgery and forecast the risk of recurrence.

7. A heart transplant is the last recourse provided no metastases.

\section{FINANCIAL SUPPORT AND SPONSORSHIP \\ Nil.}

CONFLICTS OF INTEREST

The authors declare no conflict of interest

SUPPLEMENTARY DATA (DOI)

\section{REFERENCES}

1. Mikhoparova O.Y., Tcybulkin N.A., Oshchepkova O.B. et al. Cardiac tumors in clinical practice [Opukholevye porazheniia serdtsa v klinicheskoi praktike]. The Bulletin of Contemporary Clinical 
Medicine - Vestnik sovremennoi klinicheskoi meditsiny, 2017. vol. 10, no. 4, pp. 80-86.

2. Moskvicheva M.G., Belova S.A., Kremlev S.L. Incidence of cardiovascular diseases in urban and rurral popula tion. comparative analysis [Sravnitel'nyi analiz zabolevaemosti bolezniami sistemy krovoobrashcheniia gorodskogo i sel'skogo naseleniia]. Modern problems of science and education - Sovremennye problemy nauki i obrazovaniia, 2015, no. 5, available at: https://scienceeducation.ru/ru/article/view?id=7100. (accessed 19.03.2019).

3. Storozhakov G.I. Heart Tumors. [Opukholi serdtsa] Russian Heart Failure Journal - Serdechnaia nedostatochnost', 2008, vol. 9, no. 4, pp. 194-200.

4. Sakovich V.A. Current state of the issue of surgical treatment of malignant neoplasms of the heart and pericardium [Sovremennoe sostoianie voprosa o khirurgicheskom lechenii zlokachestvennykh novoobrazovanii serdtsa i perikarda] The Siberian Medical Journal - Sibirskij medicinskij zurnal, 2004, vol. 47, no 6, pp. 5-11.

5. Nair A., Rajesh G.N., Sajeev C.G. Functional tricuspid stenosis: a rarepresentation of suspected rhabdomyoma as congenital cyanotic heart disease. Cardiology in the Young, 2017, vol. 12, pp. 808-811, doi: 10.1017/S1047951116002110.

6. Hoffmeier A., Sindermann J.R., Scheld H.H, et al. Cardiac Tumors - Diagnosis and Surgical. Deutsches Ärzteblatt International, 2014, vol. 111, no 12, p. 205, doi 10.3238/arztebl.2014.0205.

7. Acampa M., Tassi R., Guideri F. et al. Safety of intravenous thrombolysis in ischemic stroke caused by left atrial myxoma. Current Drug Safety, 2011, vol. 6, no 25, pp. 343-345, doi $10.2174 / 157488611798918665$.

8. Andrievskikh I.A., Lukin O.P., Davydov I.V. Coronary intervention on the background of the polypaty [Koronarnye vmeshatel'stva na fone polipatii]. Modern problems of science and education - Sovremennye problemy nauki i obrazovaniia, 2012, no 5, available at: http://www.scienceeducation.ru/ru/article/view?id=7171 (accessed: 19.03.2019).

9. Aravot D.J., Banner N.R., Madden B. et al. Primary cardiac tumours - is there a place for cardiac transplantation? European Journal of Cardio-Thoracic Surgery, 1989, vol.3, pp.521-524, doi 10.1016/1010-7940(89)90112-7 